CORPUS $\begin{aligned} & \text { Corpus } \\ & 12 \mid 2013 \\ & \text { Dialectologie : corpus, atlas, analyses }\end{aligned}$

La trajectoire de la dialectologie au sein des sciences du langage. De la reconstruction des systèmes dialectaux à la sémantique lexicale et à l'étymologie

Jean-Philippe Dalbera

(2) OpenEdition

Journals

Electronic version

URL: http://journals.openedition.org/corpus/2390

ISSN: 1765-3126

Publisher

Bases ; corpus et langage - UMR 6039

Printed version

Date of publication: 1 January 2013

Number of pages: 173-200

ISSN: 1638-9808

Electronic reference

Jean-Philippe Dalbera, « La trajectoire de la dialectologie au sein des sciences du langage. De la reconstruction des systèmes dialectaux à la sémantique lexicale et à l'étymologie », Corpus [Online], 12 | 2013, Online since 13 May 2014, connection on 08 September 2020. URL : http:// journals.openedition.org/corpus/2390

(c) Tous droits réservés 


\title{
La trajectoire de la dialectologie au sein des sciences du langage. De la reconstruction des systèmes dialectaux à la sémantique lexicale et à l'étymologie
}

\author{
Jean-Philippe DALBERA \\ Univ. Nice Sophia Antipolis, CNRS, BCL, UMR 7320, \\ 06300 Nice, France
}

\section{L'image actuelle de la dialectologie : la dialectologie vue de dehors}

Que représente aujourd'hui la dialectologie ? Aux yeux du plus grand nombre, peu de choses : une discipline vieillotte, passéiste, folkloriste, un savoir superflu, aucunement rentabilisable, au mieux un violon d'Ingres pour quelques originaux. Aux yeux des linguistes, une discipline mineure, l'étude de parlers locaux, le plus souvent moribonds auxquels on s'intéresse au détriment des grandes, des vraies langues : parlers locaux, l'appellation neutre de ce qui auparavant s'appelait patois est en train de prendre à son tour l'acception teintée de mépris qui caractérise ces derniers. Certes il existe encore quelques régionalistes ici et là qui tentent de brandir la dialectologie comme argument scientifique de défense de leur parler maternel ou grand maternel, mais même là le cœur n'y est pas car le dialectologue se refuse à valider les normalisations autoritaires auxquelles tentent de se livrer certains à l'intérieur des groupes de parlers régionaux. Le dialectologue ne peut accepter que l'on fasse subir aux parlers encore vivants le sort que la diffusion du français a réservé aux langues régionales. Le fait de se doter d'une norme graphique susceptible de fonctionner comme koinè écrite en vue de favoriser la diffusion et de promouvoir l'enseignement d'une langue n'est nullement répréhensible tant s'en faut, tant que l'usage parlé de cette langue demeure effectif dans sa diversité géographique. Cela devient une catastrophe linguistique si le propos 


\section{J.-P. DALBERA}

est de réapprendre «leur » langue à des générations qui ne la connaissent plus : le conflit engendré entre ce qui reste d'apprentissage passif (hérité et valorisable) et ce qui procède d'une norme externe achève le processus de mise en extinction de celle-ci. Mais alors, à quoi bon la dialectologie ? Et d'abord de quoi s'occupe-t-elle ? Il semble qu'on ne le sache plus trop. Au départ ce qui sépare la dialectologie de la philologie et plus globalement de la linguistique, c'est le fait de travailler sur l'oralité ; en liaison avec cet aspect une seconde caractéristique intervient, qui touche au mode de recueil des faits, au mode de transcription et de consignation des données linguistiques, au mode de choix des témoins consultés, bref au travail dit de terrain et à la technique d'enquête. En troisième lieu figure la prise en compte de la dimension spatiale, la variabilité de proche en proche dans le continuum géographique. Mais ni l'étude des données orales, ni l'enquête de terrain, ni la variation dans l'espace ne sont plus à même de caractériser la dialectologie : l'oralité est désormais incontournable pour la linguistique dans son entier, les sociolinguistes ont approfondi la gestion théorique et pratique de l'enquête, les géolinguistes se sont appropriés l'espace géographique... Que reste-t-il au dialectologue?

\section{L'objet de la dialectologie : retour aux sources et recentrage sur la parenté génétique}

Pour concevoir ce qui fait le propos spécifique de la dialectologie, il est utile de revenir un instant aux sources : l'intérêt qui s'est porté pendant une période sur les "parlers locaux » ne relève pas du souci immédiat de mieux les connaître. A travers eux, à travers la représentation naïve que l'on se fait de ces parlers locaux, on cherche à comprendre pourquoi les lois phonétiques ne s'avèrent que partiellement, pourquoi il $\mathrm{y}$ a toujours des exceptions : si l'on veut ne pas remettre en cause la théorie (les lois phonétiques) il faut revoir les faits, le corpus. Puisque les langues de culture ou nationales n'illustrent pas (toujours) ce que prédisent les lois, c'est que ces langues ont été manipulées artificiellement (retouchées, complétées, enrichies, codifiées... bref transformées) de telle sorte qu'elles ne constituent pas un test valide pour juger de la valeur des lois phonétiques: il 
convient donc de trouver des parlers ayant évolué de manière «naturelle », étant restés en dehors de tout interventionnisme linguistique (le bon sauvage version langue). Nous y voilà. On sait ce qu'il est advenu de cette démonstration: les parlers locaux n'ayant pas fourni la preuve que les lois phonétiques ne souffraient pas d'exception, la démonstration s'est retournée contre ses promoteurs. Bien loin de valider définitivement les lois, ce sont les aléas de leur histoire qui font les mutations des mots. La démarche a donc avorté mais elle reste révélatrice : ce qui a été tenté, avec l'essai de validation des lois phonétiques, c'est la mise en œuvre d'une vision de l'évolution linguistique dans laquelle les changements observables sont la résultante des forces de pression qui s'exercent sur la langue dans le temps et l'espace; dans le temps sous forme de vagues de mutations aveugles ne laissant à l'homme aucune prise (telles les lois phonétiques), l'espace apparaissant comme un crible qui laisse plus ou moins, plus ou moins vite, passer ces vagues de mutations et dessiner en définitive des aires (entendues comme espace ayant connu une évolution homogène). Il y a donc bien dans cette irruption de la dialectologie sur la scène linguistique l'idée sousjacente d'une corrélation étroite entre variations dans l'espacetemps et dans l'espace géographique et par ailleurs au moins l'intuition que ces deux dimensions de la variation peuvent à certains moments tirer en sens inverse et entrer en conflit.

\section{Parenté génétique et dialectes : l'approche comparatiste}

Il est temps à présent de rassembler les débris épars et d'essayer de reconstituer la poterie. Le dialectologue travaille essentiellement sur des données de l'oralité mais ne s'interdit nullement d'exploiter des données écrites quand elles existent; la dialectologie s'intéresse au premier chef à la répartition des faits dans l'espace mais cela ne lui interdit nullement de prendre en compte le temps: les cartes d'atlas sont tantôt synchroniques, tantôt diachroniques. Ce qui caractérise la dialectologie, c'est la prise en compte nécessairement conjointe du temps et de l'espace. Le doute n'est pas permis : l'objet propre de la dialectologie, c'est la parenté génétique, ou plutôt le degré de parenté génétique entre les parlers. Le terme de dialecte a été utilisé de tant de 


\section{J.-P. DALBERA}

manières différentes qu'il couvre toutes les ambiguïtés du monde ; si l'on doit l'utiliser, il convient d'être clair avec sa valeur ; dialecte fonctionne, dans notre perspective, comme un terme purement relationnel : le niçard n'est pas un dialecte mais un dialecte de la langue d'oc au même titre par exemple que le languedocien; la langue d'oc (l'occitan) est un dialecte du roman occidental au même titre que le catalan, le piémontais, le castillan ou le toscan et peu importe que certains aient acquis rang de langue nationale et d'autres pas. Puisque l'on se trouve embarqué dans une métaphore génétique, avec les désagréments que cela comporte, essayons d'en tirer avantage et soulignons que dialecte fonctionne comme cousin. Nul n'est cousin : tout ce qui peut arriver c'est que l'on soit le cousin de quelqu'un. Et chacun sait qu'il existe des degrés dans le cousinage comme il existe des degrés dans la proximité diachronique entre les parlers.

Mais dès que l'on pose que le dialectologue travaille sur un ensemble de parlers génétiquement apparentés, le plus souvent étroitement apparentés et qu'il cherche à cerner les dialectes, à délimiter les aires dialectales, on se rend compte que son objectif est de parvenir à une mesure des écarts : écarts entre les parlers modernes, écarts entre parlers modernes et systèmesource. Le mode d'opération de ces mesures, qui visent à établir la distance qui sépare chacun des parlers du système-source et sur le détail desquelles il y aurait beaucoup à dire suppose le recours au moins implicite à un arrière-plan (reconstruit ou attesté, peu importe) qui sert de repère. La meilleure mesure de la distance linguistique qui sépare le roman occidental du languedocien, de l'alpin ou du piémontais est certainement celle qui s'exprime en nombre et qualité des changements intervenus. L'étude monographique d'un parler local n'est qu'un préambule à une recherche dialectologique : celle-ci commence là où commence la comparaison. L'objet d'un travail dialectologique revient in fine à rendre compte de l'architecture d'un espace-temps dialectal à travers sa reconstruction. Le maître-mot-clé définitoire de la dialectologie est donc reconstruction. Celle-ci se décline comme élaboration hypothétique d'un protosystème et conjointement élaboration de paquets de règles ordonnées ayant pour fonction de dériver les proto-éléments (état zéro) c'est-à-dire 
pour en faire des éléments de l'état $\mathrm{n}^{\circ} 1$ qui succède à l'état zéro dans la chronologisation prise pour objet de l'étude.

\section{Un comparatiste privilégié}

Le dialectologue apparaît dès lors essentiellement comme un comparatiste, peut-être même comme un comparatiste privilégié. De fait, entre la démarche du comparatiste-dialectologue et celle du comparatiste indo-européaniste seule ou presque l'échelle change. Les problèmes que l'un ou l'autre ont à traiter sont de même nature. Mais il est vrai que les conditions de travail ne se ressemblent guère : le recul (ou l'absence de recul) par rapport aux données, des lacunes face à un déroulement continu, des univers de référence différents... Certes, le fossé est considérable mais dans tous les cas cependant l'adéquation des systèmes reconstruits à la réalité reste comparable ; ceux-ci ne sont que des systèmes intermédiaires et de pures abstractions. La reconstruction d'un ensemble dialectal se fait souvent avec des données qui ne changent pas spectaculairement d'un parler à un voisin immédiat mais qui peuvent, quand on confronte un bout de la chaîne à l'autre manifester un écart considérable. C'est alors toute une architecture sous-jacente qui se révèle et qui, dans les répétitions qu'elle affiche et les cycles qu'elle suppose, nourrit les modélisations du changement linguistique. C'est là qu'on discerne ce qui est possible en matière de langage (la vraisemblance des systèmes et des règles), c'est là qu'on touche du doigt comment des systèmes cousins que peu d'écarts séparent, confrontés à une même difficulté sont susceptibles de s'engager dans des voies diverses pour y porter remède, toutes choses qui constituent une contribution spécifique aux sciences du langage. C'est là qu'on appréhende la dynamique des mutations (gradualité vs innovation partagée). C'est là qu'il faudra puiser les modélisations du changement linguistique. Il reste néanmoins, - et cela est crucial pour notre propos -, à rappeler que, en pratique, toutes les reconstructions ne se valent pas; les plus abouties l'ont été dans les domaines de la phonologie et de la morphologie. Le lexique est beaucoup moins bien loti. Les linguistes s'accordent sur la faiblesse de ce secteur de recherche. Ainsi Swiggers dans son analyse dans une perspective épistémologique 
des principaux axes et des différents moments de la pensée étymologiste, clôt son bilan sur le «parent pauvre de l'explication étymologique : la sémantique diachronique et reconstructiviste ${ }^{1}$. Et le constat est analogue dans l'article qui ouvre le Mémoire de la Société Linguistique de Paris, consacré aux « Théories contemporaines du changement sémantique » : « la manière dont les dictionnaires étymologiques rendent compte ou tentent de rendre compte de l'évolution sémantique est profondément décevante $»^{2}$.

\section{Le parent pauvre de l'explication étymologique}

Le moment semble venu de mettre à profit des convergences entraperçues dans ce qui précède pour relancer ces laissés pour compte de la recherche que semblent être la sémantique lexicale et l'étymologie. Le nœud conceptuel pourrait être constitué par l'analyse motivationnelle et la perspective de reconstruction. Les nombreuses analyses auxquelles nous nous sommes livré dans nos travaux autour de l'ALiR et de l'ALE nous ont convaincu que des analyses fines réalisées à partir des cartes d'atlas dialectaux étaient de nature à révéler des motifs profonds, voire très profonds à la base d'unités lexicales somme toute banales. Les deux champs les plus fouillés ont été le domaine magicoreligieux ${ }^{3}$ et les zoonymes; les résultats sont très encourageants. La technique mise en œuvre consiste à se servir des motifs dégagés sur les unités lexicales transparentes comme clés pour élucider les mots opaques. Ensuite procéder à une analyse comparative selon les mêmes principes que ceux utilisés pour la phonologie. Ainsi la dialectologie livre-t-elle un corpus important et pré-formaté à l'attention des chercheurs.

\section{Le lexique peut-il faire l'objet d'une reconstruction ?}

Mais l'étymologie n'est-elle pas une reconstruction lexicale, elle qui s'autorise notamment, entre autres, à reconstruire des motsétymons quand nécessaire? Alors notre question paraît étrange

1 Swiggers (1991).

2 Rousseau (2001 : 11).

3 Voir notamment Alinei (1984). 
et fait entendre que l'on se propose d'enfoncer des portes ouvertes. Il convient donc de l'expliciter. A priori la réponse va de soi ; une démarche qui vise à identifier des formes-sources et à les relier à des formes observables en aval (quelle que soit la période considérée) ou, lorsque celles-ci n'existent pas dans les textes dont on dispose, à les re-construire (en les marquant d'une astérisque ${ }^{*}$ ) peut-elle être autre chose qu'une reconstruction? - Soit. Cette réponse pourtant n'est aucunement satisfaisante ; ce n'est pas faire preuve d'esprit byzantin que de dire qu'on est bien en présence d'une reconstruction mais non d'une reconstruction lexicale : on reconstruit la forme phonique mais on ne reconstruit guère le signifié ; or chacun sait que le signe lexical - disons ici simplement le mot - est une entité à double face signifiant / signifié.

Que la reconstruction se limite à la forme signifiante est facile à démontrer. Il suffit de feuilleter quelques pages d'un dictionnaire étymologique pour s'en convaincre. Ouvrons par exemple le $D E L F$. On y trouve toute une série d'entrées françaises, de type chèvre, doigt, maison, agneau, pierre, pain... en face desquelles figurent, avec quelques commentaires, les étymons latins respectifs : CAPRA, DiGiTUS, MA(N)SIO, -4NIS, AGNeLLUS, PeTRA, PANIS... Cet ensemble de formes ne soulève aucune difficulté. Les étymons sont là des mots latins attestés dont le référent n'a pas changé et dont la forme phonique a connu des changements bien répertoriés par ailleurs et tout à fait conformes à ce que l'on sait de l'évolution du phonétisme dans cette zone de la Romania.

\section{Le modèle proposé : principes directeurs et méthodologie}

Reculer devant la reconstruction de la face sémantique du mot dans le cadre de l'élucidation étymologique ne nous semble pourtant pas inéluctable et nous nous attacherons dans ce qui suit à montrer comment parvenir à reconstruire le signifié.

7.1 Assumer le caractère bifacial du signe et dissocier deux plans et deux analyses

Les langues naturelles se caractérisent par l'absence d'isomorphisme entre les deux plans du signe que sont l'expression et le 


\section{J.-P. DALBERA}

contenu. L'association d'une représentation à une expression s'opère dans le cadre du mot notamment où elle se fige. Mais cela n'implique nullement que les atomes constitutifs de l'un des plans soient systématiquement corrélés aux atomes de l'autre. Cette dissymétrie s'observe sans difficulté dans le changement lexical : une altération de la forme phonique d'un mot n'a pas nécessairement d'écho au plan du sens. Cela nous incite à envisager l'idée que la reconstruction lexicale passe par une double démarche : reconstruction de la face phonique d'une part, sur la base de ce que l'on sait de l'évolution du phonétisme, de la face sémique de l'autre. Le point crucial est que ces deux reconstructions doivent être conçues indépendamment l'une de l'autre ; ce n'est que par la suite qu'il faudra étudier l'interaction entre les deux, étant entendu que le changement lexical ne passe pas que par le signifiant. Il y a en effet tout lieu de croire, à voir dans la diatopie les ressorts des mutations lexicales, qu'à chaque état du système les deux faces interagissent et participent par là-même de la dynamique lexicale (attraction paronymique, pondération homonymie/polysémie...). Rééquilibrer l'étymon suppose de traiter chaque plan du signe de manière autonome. Il n'est nul besoin d'insister sur la reconstruction de la face phonique: elle est, pour l'essentiel, au point. La chose est moins claire pour la face sémantique.

\subsection{Concevoir l'analyse motivationnelle comme une recons- truction comparative du signifié}

La première phase de la reconstruction du signifié d'une unité lexicale consiste selon nous à mettre en évidence le ou les motif(s) qui constituent le fondement de la création de celle-ci et à tenter de cerner les transformations que ce motif est susceptible d'avoir connues. Certes il ne s'agit pas seulement de piocher dans un sachet de motivations superficielles apparentes. Pour accéder à ces motifs, il faut tirer les conséquences du fait que le langage est son propre métalangage, qu'un mot se glose par un autre mot ; cela implique, en l'occurrence, que le signifié d'un mot ne peut être communiqué que par le biais de l'un (ou de plusieurs) de ses équivalents approchés, c'est-à-dire toujours par le biais d'autres mots. L'idée, à partir de là, est d'utiliser les manifestations concrètes du sens que sont les diverses expressions 
qui lui sont associées pour approcher et reconstruire le sémantisme qui les sous-tend. Cela revient à traiter les signifiés selon une technique de reconstruction comparative bien rôdée sur le plan de l'expression. De la même façon que le linguiste est capable de reconstruire un proto-phonème à partir des divers aboutissements que celui-ci connaît dans des contextes différents ou dans des idiomes différents, de même, le linguiste, prenant en compte la variation lexicale se met en mesure de reconstruire la source sémantique d'où se déduisent les différentes manifestations sémantiques observées (et, complémentairement, de maîtriser les changements de sens). Il ne lui reste alors, pour progresser dans une recherche étymologique, qu'à répondre à la question : les autres dénominations - opaques - s'éclairent-elles si on leur associe un signifié comparable à celui précédemment trouvé ?

Deux points majeurs, par conséquent, se font jour dans notre approche : d'une part, la matérialisation du sens sous la forme d'un paradigme de variantes lexicales interprétées comme quasi-équivalentes dans leur emploi ; d'autre part, la technique éprouvée de reconstruction comparative. Et le support concret de la réflexion pour mener à bien ces deux manœuvres peut être, dans un premier temps du moins, un objet tel qu'une carte onomasiologique d'atlas dialectal. Le signifié (profond) n'est que sporadiquement accessible sous la forme d'un motif de manière immédiate : le motif doit le plus souvent faire l'objet d'une reconstruction et la reconstruction de celui-ci n'est convaincante et ne se valide que si elle entraîne en retour la mise en lumière de nouvelles expressions de ce motif. La recherche motivationnelle consiste donc à exploiter les équivalences d'emploi pour accéder aux équivalences de représentation ${ }^{4}$. Et il faut noter qu'elle a ceci de particulier qu'elle engendre naturellement sa propre évaluation: le chercheur se rend compte très vite si sa clef ouvre des portes oubliées ou que l'on croyait condamnées.

4 Nous nous trouvons là dans une position qui rappelle beauoup celle que préconise Benveniste (1969 I. :12); il s'agit, écrit celui-ci « par la comparaison et au moyen d'une analyse diachronique de faire apparaître une signification là où, au départ il n'y a qu'une désignation ». 


\section{J.-P. DALBERA}

\subsection{Postuler la continuité lexicale dans l'espace et le temps}

Il ressort de ce qui a été établi plus haut que la dynamique lexicale est à l'œuvre en permanence, que, pour dire les choses autrement, le cheminement des mots est cyclique (motivationconvention-arbitraire) $)^{5}$. Cette propriété pourrait bien ouvrir des horizons intéressants pour la reconstruction lexicale. S'il est vrai que la remotivation est à la fois potentielle en permanence et susceptible de s'effectuer de plusieurs manières différentes, il devient crucial d'examiner de près les avatars motivationnels. Une remotivation peut s'opérer à l'identique si le motif d'origine est toujours aussi prégnant dans l'imaginaire collectif ou se renouveler si la représentation qu'on se fait de tel ou tel pan de réalité change. Mais en tout état de cause, elle donne des clefs pour accéder tant aux représentations diverses qu'une communauté linguistique se fait du réel, qu'aux mécanismes proprement linguistiques qui autorisent ou favorisent le changement de représentation. Une conséquence importante est que l'argumentation étymologique doit être conçue comme achronique et atopique ; seuls les états du lexique résultants sont à envisager situés dans l'espace et le temps ; ils constituent alors des équilibres instables et fragiles.

7.4 Postuler une homogénéité de représentation par défaut à l'intérieur d'une aire linguistique génétiquement délimitée

La proposition ci-dessus en 7.3 doit être complétée par un postulat d'homogénéité minimale des faits linguistiques qui consiste à supposer que les motifs des représentations du réel que véhicule le langage ne sont ni individuels ni anecdotiques; en d'autres termes que la vision du monde d'une communauté à un moment donné est pour l'essentiel homogène ; il s'agit presque d'une proposition tautologique. Il faut done s'attendre, pour ce qui nous concerne ici, à trouver, quand on travaille sur des périmètres restreints, des motifs de création de signes récurrents et c'est cette permanence dans la représentation qui incite à y voir une sorte de sémantisme commun ; celui-ci dès lors est candidat au statut de fondamental. Encore faut-il que ce point soit bien interprété : nous voulons dire que l'homogénéité de motif doit

5 Sur cette notion de cycle motivationnel, cf. Dalbera (2006a) : 23-27. 
être envisagée par défaut comme le caractère non marqué de la situation. Cela n'empêche nullement qu'il y ait des cas où une diversité notable se manifeste. Par exemple quand les motifs traduisent l'empilement de plusieurs strates linguistiques, la vision en à-plat de l'aire ne renvoyant alors qu'à une hétérogénéité factice, artefact du regard porté. Dans ce cas, on a affaire à une situation marquée.

\section{Illustrations}

Quelques illustrations sont indispensables pour mesurer l'écart qui sépare la reconstruction lexicale ainsi initiée de la démarche étymologique classique. Il est important entre autres de voir à l'œuvre la mise en évidence des motifs, les notions de continuité de représentation, d'homogénéité en périmètre restreint et de récurrence. Le premier exemple traité concernera les noms du martinet ${ }^{6}$.

\subsection{Les dénominations du martinet (d'abord en pays langue- docien puis au delà)}

Le martinet, comme chacun sait, est une espèce d'hirondelle. Sa dénomination scientifique est Apus apus. Dans les parlers populaires il reçoit, selon le témoignage de la dialectologie, une multitude d'appellations plus étranges les unes que les autres. Nous nous sommes posé la question d'un éventuel motif qui serait à la base de quelques-unes au moins de ces appellations. Nous n'avons pas été déçu. La liste des types d'appellations que l'on peut trouver en consultant notamment les atlas linguistiques $\mathrm{du}$ monde roman se présente comme suit : martinet, martelet, foucil, balestrièr, barbajòu, barbiròu, rascle, rudilou, issòlo, passovolan, trenquet, sbiro, passeru volan ${ }^{7}$ pour le sud de la France, à quoi s'ajoutent des dénominations recueillies en terre

6 Le lecteur trouvera l'analyse des dénominations du martinet dans plusieurs textes, notamment Dalbera $(2002,2006)$.

7 Tous ces mots s'emploient pour désigner l'oiseau martinet. La traduction littérale des mots de cette série serait la suivante : «martinet», «martinet », « petit marteau », « arbaletier », « faucille », « barbier », « sbire », « sorte d'ogre » ou « petite bestiole ». 


\section{J.-P. DALBERA}

espagnole : vencejo, ou italienne : rondone, balestruccio, barbaottu, sbiru... La liste n'est évidemment pas exhaustive.

Importante et curieuse diversité. En première approximation, ces dénominations n'offrent ni unité ni cohérence ; qu'y at-il de commun à un martinet, une faucille, la barbe de Jupiter, un oiseau qui vole (sic), un soudard... ? Ce bric à brac lexical prend néanmoins un relief certain dès que l'on a bien voulu gratter la mince couche superficielle. Tout tourne, semble-t-il, autour de deux images.

Le martinet en vol, d'une part, évoque (irrésistiblement, semble-t-il) par sa silhouette l'arbalète et les traits qu'elle décoche; d'où ces dénominations récurrentes de type balestrièr, aubarestier, balestruccio ; cette métaphore est également, de manière moins immédiatement visible, à la base de sbiro: les sbires (aujourd'hui hommes de main chargés de la basse besogne) sont d'abord dans l'Italie du quattrocento des gens d'armes au service des princes, appartenant à des compagnies d'archers ou d'arbalétriers; l'image ne se dément pas et l'on pourrait ajouter d'autres appellations analogues du martinet comme, par exemple wallon aîrchi «l'archer ». Les ailes déployées du martinet en vol, alliées à la rapidité et au caractère rasant de celui-ci font penser aussi au geste du faucheur et au mouvement des lames recourbées d'outils agricoles, de type faucille : c'est la source de bon nombre d'appellations, telles fauç, fauçil mais divers autres outils peuvent faire l'affaire, une fois la matrice métaphorique en place ; ainsi s'expliquent des dénominations comme trenquet qui désigne, entre autres, une serpette de vendangeur, eissòla qui renvoie à une petite hache courbe $(T D F)$ ou encore martelet qui désigne une sorte de petit marteau... Cela nous permet en outre de comprendre ce nom de volan qui est attesté en maints endroits : il ne s'agit évidemment pas d'un mot construit sur « voler » (le trait ne serait guère discriminatif pour un oiseau) mais de volam, autre terme en usage désignant la faucille. Et de même que les dénominations forgées sur arbalète campent un oiseau-arbalète ou un arbalétrier, celles qui sont liées à la faucille campent un oiseau-faucille (littéralement passo volam) ou un faucheur. Et c'est à cette caractéristique de vol rasant que l'Apus apus doit certaines de ses dénominations 
parmi les plus étranges : c'est un raseur que l'on dénomme donc barbier, barbirou, rasclaire ${ }^{8}$ ou même, avec dérapage, barbajou.

L'analyse comparative des variantes de dénomination de l'Apus apus conduit donc à un motif majeur, un sémantisme fondamental : la représentation que les gens se font du martinet est celle d'un faucheur, d'un raseur (homme ou instrument). Reste à savoir quelles sont la validité et l'extension de cette représentation. L'analyse qui précède a livré une clef. Celle-ci pourraitelle faire office de passe-partout? Un rapide élargissement du champ d'étude livre des confirmations au delà de toute espérance. Bon nombre de formes d'Italie septentrionale désignant le martinet sont considérées comme d'étymologie obscure ; elles semblent s'égrener dans l'espace sans principe organisateur. Il est vrai que la destructuration phonétique de ce terme et son émiettement en variantes innombrables ne facilitent pas une appréhension cohérente et univoque des faits. Un usage immodéré de la métathèse notamment, combiné à des déplacements d'accent (cf. [si'zile], ['siliga]) rendent délicat l'établissement de la chaîne des formes; de telle sorte que chacun y va de sa proposition étymologique et que l'on rivalise d'intuition et d'astuce : Bartoli (1945 : 39) pose une base CICINULA en se fondant sur une définition du Corpus glossariorum latinorum: Hirundo nomen passeris cicines; Zamboni (1972: 239) propose une base (AVIS) CILICA, l'oiseau de Cilicie qu'il considère comme «foneticamente ineccepibile (...) e semanticamente plausibile se si pensa alle caratteristiche migratorie di questi ucelli $»^{9}$. Ces essais, fort louables au demeurant, pèchent par leur caractère non systématique qui les rend non falsifiables. Est-il imaginable de voir dans ces dénominations les images-sources prégnantes, la métaphore créatrice majeure de l'arbalétrier ou du faucheur? A priori il semble que la réponse soit négative en ce qui concerne les formes dialectales italiennes; mais, à la réflexion, si l'on prend en considération le stade latin, l'hypothèse prend corps : il existe en effet dans cette langue un mot S3C3LIS qui

8 Littéralement « « racleur »; mais l'image prend d'autant plus de consistance que le ramoneur se dit rasclo chamineio et que le martinet est souvent désigné comme l'hirondelle de cheminée.

9 Cf. Martins \& Vitorino (1984 : 144). 


\section{J.-P. DALBERA}

désigne la faucille (S3CIL3RE «faucher») et qui, au plan phonique, sous-tendrait sans difficulté les réalisations dialectales dans toute leur diversité ; [si'zile] et alii ne seraient donc autres que des continuateurs d'un mot latin qui désigne la faucille. Certes les perturbations qui affectent le signifiant bousculent cet ordonnancement et gomment le lien génétique mais guère plus qu'en Languedoc où faucilh peut dériver vers un énigmatique forsit et où balestrièr se déforme en vulastrièr. L'hypothèse étymologique est donc cette fois issue d'une suggestion sémantique, il convient de le noter ; ce n'est pas un mot latin qui sert de source partagée aux formes languedociennes et frioulanes, c'est une image-dite $e^{10}$ formulable par des mots différents, c'est-à-dire une représentation exprimée. Une situation complexe se présente également à l'ouest du Languedoc. En Espagne, la forme vencejo qui désigne l'Apus apus laisse les analystes perplexes ; néanmoins le poids du sémantisme mis en lumière par la comparaison incite Corominas ${ }^{11}$ à rattacher vencejo à FALCILLU malgré les difficutés phonétiques. ${ }^{12}$ La récurrence motivationnelle n'est donc nullement argument négligeable aux yeux des maîtres de l'étymologie.

Nul ne contestera, pensons-nous, le caractère récurrent de la représentation en même temps que la plasticité de celle-ci. L'image peut se modifier quelque peu, tout comme la forme phonique signifiante, évoluer sans pour autant devenir illisible, ce qui revient à dire que signifiant et signifié mènent alternativement le jeu, que l'un comme l'autre sont susceptibles d'évoluer et surtout que ces évolutions sont autonomes, s'opèrent parallèlement ${ }^{13}$. L'équilibre se rétablit dans la reconstruction entre dynamique phonique et dynamique sémique.

Enfin, un coup d'œil du côté du grec nous apprend que la la faux (drépanè) et le martinet (drépanis) portent dans cette langue quasiment le même nom. La preuve est faite qu'en

\section{Parallèle à lieu-dit.}

$11 \mathrm{Au}$ terme d'une analyse comparative, précisément, cf. $D C E C H$.

12 Pour une analyse plus complète, cf. Dalbera (2006a :109-125).

13 Le cas où elles en viennent à converger constitue l'attraction paronymique ; mais ce n'est pas le cas général. 
matière de lexique, la reconstruction sémantique opérée à partir d'une analyse comparative de dialectes modernes est de nature à livrer des résultats qui dépassent largement le périmètre du corpus à partir desquels l'analyse a été élaborée, et notamment d'excéder le cadre synchronique. Prenons donc acte du fait que le signifié premier est une primitive logique et non chronologique. Et de ce que la possibilité de faire des prédictions (éminemment falsifiables) permet de mesurer la fiabilité et la pertinence des hypothèses avancées. On aura remarqué au passage le rôle majeur tenu par la dialectologie dans cette démarche. Elle seule est en effet de nature à proposer un corpus suffisant pour suggérer puis asseoir des hypothèses sémantiques ; ce n'est qu'à travers le foisonnement des variantes se complétant et se corrigeant l'une l'autre que la reconstruction s'est avérée autorisée. Le même raisonnement, appuyé sur les seules formes des langues nationales, aurait certainement eu du mal à emporter la conviction.

\subsection{Les dénominations des oiseaux de types grives ou merles}

Notre deuxième illustration concerne encore des noms d'oiseaux. Il s'agit cette fois des noms populaires des turdidés, c'est-à-dire des grives auxquelles nous adjoindrons les étourneaux. Les parlers de France, au delà de quelques mots onomatopéiques tels tyatya ou chacha, connaissent, considérées en vrac, les formes suivantes ${ }^{14}$ : grive, merle, draine, litorne, mauvis, tourd, étourneau, sansonnet, oc. tordol, trida, quina, cesera...; les parlers d'Italie attestent des types correspondants: turlu, sturnello, merula, marvissi... et y ajoutent quelques autres: turdena, sassello... Peuvent encore y être adjointes quelques formes relevées dans la péninsule ibérique par exemple, ou en Dacie : $s t u r d z$. Il y aurait certes beaucoup à dire sur la liste ainsi dressée : ces noms renvoient à diverses espèces de grives ou à des génériques variables selon les régions, prennent ou non en compte la distinction mâle / femelle, cèdent parfois la place à des appellations sous forme de gloses : tordo reale, grive de

$14 \mathrm{Il}$ s'agit là des types lexicaux. Pour une analyse plus précise incluant les variations, on se réfèrera à Dalbera (2009a) et (à paraître). 


\section{J.-P. DALBERA}

pays, merle noir ou encore grive de vignes ; mais il n'est guère possible dans le présent cadre d'exposer la question en détails ${ }^{15}$.

\section{Reconstruction autonome de la face sémantique et étymologie}

Nombreux sont les cas où la démarche étymologique classique tourne court, une fois la constatation faite que la forme phonique est opaque et qu'il n'existe pas en latin de mot susceptible de sous-tendre cette forme phonique moderne. Le recours à une reconstruction du sens permettrait de démontrer, si celle-ci aboutit, que les deux reconstructions sont effectivement autonomes et que la reconstruction lexicale ne peut faire l'économie ni de l'un ni de l'autre plan. Nous nous efforcerons dans ce qui suit de tirer la leçon de la confrontation des résultats que produit l'étymologie classique à ceux qu'autorise une reconstruction lexicale au sens où nous l'avons définie. Nous examinerons à cet effet le cas des différents noms des grives et des merles dans les langues et parlers romans ${ }^{16}$. Que nous apprend sur ces noms la consultation des dictionnaires étymologiques? Pour ce qui concerne le français, les sources étymologiques présentées comme sûres se résument à peu de choses : merle continue lat. MeRuLA; de nombreuses formes gallo-romanes, de type oc. ['turt], continuent par ailleurs TuRDUS ou l'un de ses dérivés, par ex. TuRDuLUS $>$ oc. ['turdu]. Ces deux bases se retrouvent dans toute la Romania. L'appellation grive, en revanche, est davantage localisée (gallo-roman) et a fait l'objet d'une controverse ; certains la rattachent à un adjectif exprimant la provenance de ce migrateur : ce serait GRAECUS, l'oiseau qui vient de Grèce, griva, gribo représentant la forme féminine de grieu " grec »; d'autres y voient « l'oiseau tacheté », « l'oiseau criblé », gribo continuant une forme nominale construite sur CRIBR1RE «passer au crible, tamiser $»^{17}$. C'est à peu près tout. Le bilan

15 On pourra se référer, pour une étude détaillée à Dalbera (2010) et Dalbera (à paraître).

16 Ce problème a fait l'objet d'une conférence (Madrid 2007). Le texte est à paraître, accompagné de cartes dans le volume 3 de l'ALiR signalé dans la note qui précède.

17 Guiraud (1967 : 40-50). 
étymologique est néanmoins vécu comme satisfaisant puisque trois étymons sont identifiés TURDUS, MERULA et GRAECU, -A et attestés en latin. En outre l'évolution qui les mène jusqu'à fr. tourd, merle, grive, à l'it. tordo, merula ou à des dérivés tordena, tordola, à l'esp. tordo, merlo ne pose pas de problème grave. Seul le rapport qui relie grive à sa source n'est pas immédiat ; mais dans l'ensemble, le contrat étymologique, s'il est vrai qu'il se limite à identifier la source latine, à pointer des jalons historiques de la continuité de sa forme phonique et à s'assurer qu'il n'existe pas de rupture brutale entre ses emplois latins et ses emplois modernes est rempli ${ }^{18}$. On remarque néanmoins que les laissés pour compte sont nombreux ; on ne sait que peu de choses des sources des appellations françaises draine, litorne, mauvis ni d'appellations italiennes comme sassello ni $a$ fortiori d'appellations dialectales comme oc. trida, turdre, cesèra, cors. tridzina... pour ne mentionner que les plus courantes; et le peu que l'on «sait» est trop anecdotique pour n'être pas décevant : autant de principes explicatifs différents qu'il y a de cas d'espèces. Ainsi le tordo sassello serait « la grive de roche » (sassello continuerait SAXATILE); pourquoi pas ? Mais encore... Le nom de la trido serait onomatopéique : il est évident pour tout le monde que cette grive-là émet un son [trtr] ; le nom du turdre procèderait d'un croisement avec celui de la tourterelle (TURTUR)... Et même si l'on admettait de pareilles formessources, on n'aurait pas progressé d'un iota sur le signifié des noms de ces oiseaux. Benveniste (1966), dans les Problèmes sémantiques de la reconstruction, s'est attaché à dénouer les fils d'un problème du même genre, concernant les dénominations latines du cochon. Essayons de voir comment il a posé le problème. Dans le développement qu'il consacre à SUS et PORCUS, Benveniste conteste l'emploi attribué traditionnellement à ces deux mots, à savoir que SUS désigne l'animal sauvage tandis que PORCUS est réservé à l'animal domestique. La discussion dans laquelle il s'engage n'est donc pas complètement comparable à celle qui nous occupe dans la mesure où elle n'est pas à

18 On sait par ailleurs qu'il est assez courant de désigner un animal (ou un produit) à l'aide du nom de la contrée d'où il provient. 
proprement parler, étymologique. Elle ne remet nullement en question l'existence d'une base étymologique SU- distincte d'une base PORCO- dont on retrouve des continuateurs tant pour l'une que pour l'autre, dans la plus grande partie du monde indo-européen. Toutefois ce que l'analyse révèle à ses yeux le conduit à mettre en doute les inférences qui découlent de ces emplois respectifs de SUS et PORCUS en matière de realia du monde IE, plus précisément de la domestication du porc dans cet espace et de la chronologie relative des faits.

Essayons d'établir ce qui est pertinent pour nous, à savoir sur quoi le raisonnement benvenistien s'appuie. Trois ordres de faits sont tour à tour allégués. Le premier résulte d'un examen minutieux des emplois respectifs de ces deux termes dans les textes et de l'éclairage contextuel éventuel. Il ressort de là, selon Benveniste, que SUS peut tout aussi bien que PORCUS désigner l'animal domestique. Celui-ci note alors que, s'il s'avère que SUS et PORCUS y sont effectivement devenus synonymes, le lexique latin se fait indûment redondant sur ce point. Ce qui conduit à une autre lecture du couple SUS/PORCUS en l'occurrence adulte/petit. Mais laissons le résultat pour focaliser sur la nature de l'argumentation. En quoi consiste ce contexte d'emploi ? Le contexte allégué peut être simplement l'entourage syntagmatique immédiat; par exemple le fait que l'animal de lait est dit lactens porcus mais jamais *lactens sus. Il ne se limite néanmoins pas à cela; il est fait aussi de corrélations. Benveniste remarque dans un chapitre du De Agricultura de Caton où se trouve décrit un protocole de sacrifice que «le sacrifice de fait comprend trois animaux, porcins, ovins, bovins mais si l'on compare les termes du sacrifice nominal: sus, ovis, taurus et ceux de l'offrande réelle : porcus, agnus, vitulus » on se rend compte que la distinction sus/porcus est du même ordre que celle que l'on observe dans ovis/agnus et taurus/vitulus à savoir adulte/petit. Enfin peuvent être mises à contribution des gloses ; on lit ainsi chez Varron : «porcus graecum est nomen... quod nunc eum vocant khoîron ${ }^{19}$.

19 Benveniste (1969 : 29-30). 
Laissons là le cas d'espèce et les détails. Le lecteur aura remarqué qu'on se trouve en présence dans l'argumentation sémantique de trois ordres de faits correspondant à des propriétés que le dialectologue connait bien parce qu'il les rencontre dans l'espace en permanence : ce que nous avons appelé 'la création lexicale par spécification d'un générique' (qui débouche sur des oppositions privatives), la valeur structurale (qui procède des relations paradigmatiques oppositives) et 'l'équivalence approximative' (en acte dans toute traduction ou reformulation).

Ces propriétés sont à considérer à la fois dans une perspective synchronique et dans une perspective diachronique et éclairent chacune un pan du mécanisme de changement lexical. Qu'un besoin de distinction se manifeste, au terme générique s'adjoint une caractéristique : TURDUS, TURDUS MERULA. Que nous apprend une approche comparative du tissu dialectal moderne ? La difficulté est-elle moindre in situ ? Ne peut-on esquisser une représentation tant soit peu stable de ces oiseaux ? Que peuvent bien signifier ces mots trida, cesèra, sassello ? Guiraud a avancé l'idée, comme on vient de le voir, que la grive n'était autre qu'une « tachetée », une " criblée ${ }^{20}$, ce en quoi il rejoint bien des impressions ou commentaires de connaisseurs ${ }^{21}$ : « les grives ont une moucheture particulière à laquelle les oiseleurs ont donné le nom de grivolure $»^{22}$; d'autres témoignages présentent spontanément griolé comme équivalent de grivelé et de marbré ; ou encore grivoté comme équivalent de grivelé et de tacheté. La piste mérite donc indéniablement d'être suivie. Et très vite on se rend compte, à y regarder de plus près, que grive n'est pas la seule dénomination à s'articuler sur cette représentation. Les découvertes ne traînent pas : en premier lieu lat. TR3TUM est le supin d'un verbe TEReRE qui signifie « trier », plus précisément « frotter» (pour polir, pour séparer la balle du blé.... $)^{23}$; une trida (variante trio) ne serait-elle pas TR3TA une «triée », un genre de «passée au crible»? En

20 Guiraud (1967).

21 Cf Guiraud (1967 : 30-40)

22 Toussenel in Rolland (1967) : 234.

23 Il s'agit de la racine sur laquelle est forgé TRIBULUM > cors. tribbiu. 


\section{J.-P. DALBERA}

second lieu le sas (source latine SAETACIUM) «tissu de crin ou de soie entouré d'un cercle de bois qui sert à passer de la farine ou des liquides » n'est autre en afr. qu'un genre de tamis ou de filtre; sasser signifie «passer au sas, au tamis $»^{24}$; it. sassello ne serait-il pas un sassé, un genre de "criblé » ou de « tamisé » ? Et la cesèra ne serait-elle pas aussi une « tachetée », avec l'indication d'une tache de forme particulière, évoquant un pois chiche si l'on admet que ce mot repose sur une source latine $\mathrm{CiCeR}(\mathrm{A})$ " pois chiche »? ne serait-elle pas la « grive à pois »? Coïncidences ? Si un doute subsiste, il est aisé de le balayer, en montrant que ce jeu de représentations est récurrent. Il existe ainsi en français un autre couple nom-verbe dénominal équivalant à crible-cribler, passe-passer, sas-sasser, trie-trier : c'est tamis-tamiser (source STAMEN « chaîne [du métier vertical des tisserands anciens] », au pluriel étamines ; étamine est donné en afr. avec le sens de "tamis » $(D A F)$; or il existe en langue d'oc un oiseau nommé tamisièr; ce n'est pas une grive mais un oiseau de proie, un genre d'épervier ou d'émouchet, en d'autres termes un oiseau moucheté et cet épervier a pour nom dans la région de Nice l'escrivèu, qu'il faut entendre non pas comme en rapport avec " écrire » mais de nouveau comme le criblé (sur CRIBELLUM); son nom dans l'Isère est d'ailleurs la criblette. Cela ne s'invente pas : les faits sont massifs. Or ces propriétés sont autant de dimensions de la variation qui constituent l'ordinaire du dialectologue. Ce sont là des données incontournables en dialectologie et qui mériteraient d'être intégrées dans une modélisation du langage et de son développement. A ces variations sur l'image-source ne manquent pas de faire écho des variations des formes phoniques-sources complètement indépendantes ${ }^{25}$ pour lesquelles nous renvoyons le lecteur à Dalbera (2010). Une fois que l'on maîtrise le sémantisme fondamental et la matrice morphosémantique, on se rend compte que bon nombre d'appellations étranges se révèlent de fait banales, répétitives, mettant en jeu les mêmes ressorts mais ont été rendues

$24 \mathrm{Cf}$. encore fr. ressasser (= « trier et retrier ») une idée.

25 Par exemple des fausses-coupes (litorne : étourneau, quino: turquino), des éléments adjoints (sassello : sansonnet), etc. 
méconnaissables à l'issue d'une sorte de balayage aléatoire effectué par la convention.

\section{L'intégration et le redéploiement des dénominations dans le cadre de champs lexicaux instables}

Une troisième dimension est en effet à prendre en compte dans un essai de reconstruction lexicale, dimension caractéristique et définitoire du langage : le caractère structural. Les dénominations ne s'empilent pas n'importe comment mais se composent, organisant ou ré-organisant temporairement des champs. A l'intérieur de ces champs jouent des distinctions, des oppositions comme dans l'univers phonématique : les unités se définissent négativement les unes par rapport aux autres, par opposition. On vient de voir un exemple caractéristique : le merle, en latin, dénommé "l'uni, le pur, le sans-mélange », l'oiseau au plumage uniforme ; cette appellation ne prend véritablement sens qu'à l'intérieur du domaine de définition des turdidés : toutes les grives sont tachetées sauf le merle. La pression paradigmatique se fait sentir d'emblée. Et ces champs sont susceptibles de se transformer à la suite d'une modification de telle ou telle de leurs unités constitutives (sortie d'usage, opacification, remotivation...). Il en résulte une dissociation du signe linguistique par rapport au référent qui parachève le processus d'arbitrarisation du signe. Le premier degré consiste, on l'a vu, en une inversion des rapports entre motivation et usage ; la convention d'usage prend progressivement le pas sur la motivation créatrice d'origine et finit par se substituer à elle. Le deuxième degré consiste en ce que le réseau de relations qui s'est constitué autour d'une notion et qui structure le champ lexical de celle-ci est l'objet, au cours de l'évolution, de métamorphoses consécutives notamment à l'introduction de signes nouveaux. L'entropie consécutive à leur irruption dans le champ engendre des réactions qui peuvent devenir des réactions en chaîne, par exemple un processus de type chaises musicales. Ce qui doit être souligné ici, c'est que le désordre créé par les réaménagements dans le cas de systèmes phonématiques prend une tout autre ampleur dès lors qu'on a affaire à des unités significatives. Que l'effet produit soit dans le premier cas, une « inversion de timbre» ou 


\section{J.-P. DALBERA}

une perte de distinction de degré d'aperture cela interpelle le linguiste, cela peut intriguer quelques locuteurs perspicaces, mais cela n'a pas d'incidences autres. Tandis que lorsque c'est l'emploi et notamment la fonction référentielle qui est touchée, les choses sont pour les locuteurs beaucoup plus difficiles à admettre.

Tous ceux qui ont la curiosité de feuilleter des cartes d'atlas dialectaux sont surpris par la nature des dénominations (très variées) que connaît, par exemple tel ou tel animal. Deux points surtout les intriguent : le caractère « extravagant» de certaines dénominations et le caractère "contradictoire» de certaines autres. Le fait qu'un serpent comme l'orvet soit appelé ici ou là le serpent ver, le ver aveugle ou l'anguille de terre n'épate personne; des appellations de ce même serpent comme salamandre ou scorpion (andalou [ehkur'pjon]) surprennent déjà davantage mais on reste dans le domaine du pensable car on pressent qu'il pourrait y avoir un trait en partage (le caractère dangereux ou venimeux par exemple) qui justifierait la métaphore ; mais quand cela devient [anvj'o] soit « vieil âne » comme cela arrive en certains points de l'aire dialectale francoprovençale ou cabra cega soit " chèvre aveugle " en certains points dialectaux portugais ou encore aniel soit « agneau » en certains points dialectaux catalans... on sort des limites de l'épure. C'est au cours de ces redéploiements que ces appellations déconcertantes apparaissent : les mots se repositionnent les uns par rapport aux autres, se réévaluent à l'aune des autres tandis que les référents demeurent. Il s'ensuit que la motivation d'un mot, si elle est restée perceptible, peut se trouver en porte-à-faux par rapport au référent : une poule, c'est initialement une petite (PULLUS, -A), un rejeton de gallinacées, l'adulte étant la géline (GALL3NA); mais si le champ se recompose avec notamment l'apport de poulette ou poussin (PULLICENUS), la petite (PULLA) se mue en fille-mère et en vient à se substituer à la géline dans son rôle de pondeuse et de couveuse. Bref, la poule réfère à la mère tout en signifiant l'enfant.

Ce troisième temps de mise sous tension de la structure lexicale, après la création sur fond de représentation motivée de l'univers et l'appropriation collective sous forme de convention sociale d'usage n'est pas le moins important tant s'en faut. Il est 
rendu particulièrement difficile à maîtriser par le fait que le paradigme dans lequel s'installent les signes nouveaux-nés est flou. Le jeu des oppositions n'est opératoire que lorsque les virtuels sont bien définis. Or on sait bien que les savoirs des locuteurs varient considérablement de l'un à l'autre. Un chasseur sera tenté de distinguer dans son tableau de chasse une draine d'une litorne ou d'un mauvis; mais la plupart des citadins n'y verront que des grives et ce dans le meilleur des cas ; le berger sera attentif à l'âge de ses bêtes et se gardera de mettre dans le même sac l'anounge (la bête de l'année), la bima, celle qui a deux ans ou qui a porté deux fois et les autres mais les autres locuteurs ne verront dans tout cela que des moutons; le petit prince de Saint-Exupéry dit: " dessine moi un mouton!"», et non un mouton d'un an. Le terme dit générique s'introduit ainsi dans le jeu lexical, générique relatif : draine, litorne, musicienne, mauvis à un niveau, subsumés par grive mais, à un autre niveau, grive, merle, étourneau subsumés par passereau etc. Par ailleurs, le générique est censé être le prototype ; mais le référent à quoi renvoie le terme qui sert de prototype n'est pas constant, n'est pas le même partout ; LA grive (tout court) c'est ici la musicienne, mais là-bas la draine qui s'est sédentarisée, qui est devenue la "grive de chez nous" lig. turlu nostral. Il n'en reste pas moins que les locuteurs savent confusément qu'ils ne savent pas, qu'ils ne connaissent que très peu les grives mais que d'autres savent, qu'il y a donc un univers de référence dans lequel ces oppositions jouent; ils n'ignorent pas que le paradigme n'est pas clos.

Récapitulons. Les spécificités de la reconstruction lexicale par rapport à l'étymologie classique peuvent donc se résumer comme suit :

- La reconstruction lexicale s'opère indépendamment sur l'un et l'autre des deux plans du signe ; ce faisant, jouant sur deux claviers au lieu d'un, elle multiplie les chances d'élucidation des cas dits obscurs et donne une assise plus solide aux résultats obtenus. Une telle mise à égalité du signifié et de la forme phonique dans l'investigation étymologique revient à réconcilier approches onomasiologique et sémasiologique. 


\section{J.-P. DALBERA}

- Elle est fondamentalement comparatiste, logiquement achronique et atopique mais reste malgré cela profondément ancrée dans le linguistique du fait de la masse de faits triés et traités au cours de la phase préliminaire; le socle d'hypothèses motivationnelles est solidement enfoncé dans le terreau dialectal.

- Elle est structurale et conçoit les états successifs du système lexical comme autant de systèmes spécifiques.

- Elle ne renonce nullement à l'étymon, mais fait de celui-ci un mot-jalon matérialisé doté d'une forme phonique et d'un sens corrélé.

- Elle est falsifiable dans la mesure où elle prédit la récurrence : il est donc aisé d'établir si les sémantismes fondamentaux qu'elle croit pouvoir proposer gardent une validité en dehors des aires géographiques ou des époques où elles ont été avancées. Rien n'interdit de mettre au banc d'essai d'autres systèmes linguistiques extérieurs au périmètre de l'étude initiale les hypothèses sémantiques et phoniques.

La première page de ce texte laissait voir une discipline dialectologique défaite, moribonde, rognée de toutes parts par ses voisines ; au terme de sa trajectoire elle apparaît ragaillardie et ayant retrouvé une place de choix au sein des sciences de langage ; c'est elle en effet qui relance et supporte la sémantique lexicale (reconstruction du signifié à partir du motif utilisé comme clef) ; c'est elle qui lance l'idée d'une double reconstruction des deux plans du signe (effectuée de manière autonome sur chacun des deux plans); c'est elle enfin qui, à travers le caractère alternatif du changement lexical tantôt initié sémantiquement, tantôt impulsé phoniquement, jette les bases d'un lexique conçu comme le croisement de la sémasiologie et de l'onomasiologie.

\section{Références bibliographiques}

\section{Dictionnaires et Atlas}

ALE : (1986-). Atlas Linguarum Europae. Assen-Maastricht, Van Gorcum, puis Roma : Istituto Poligrafico e Zecca dello stato.

ALiR : (1996-). Atlas Linguistique Roman. Roma : Istituto Poligrafico e Zecca dello stato. 
DCECH : Corominas J. \& Pascual J.A. (1980-91). Diccionario Crítico Etimológico Castellano e Hispánico. Madrid: Gredos.

DELF : Bloch O. \& Wartburg W von (1950-57). Dictionnaire étymologique de la langue française. Paris : PUF.

TDF : Mistral F. (1978). Lou Tresor dòu Felibrige ou Dictionnaire Provençal-Français. Aix-en-Provence.

\section{Ouvrages et articles}

Alinei M. (1980). «The structure of meaning revisited», Quaderni di Semantica I : 289-305.

Alinei M. (1984). Dal totemismo al cristianesimo popolare. Sviluppi semantici nei dialetti italiani ed europei. Alessandria : Edizioni dell'Orso.

Alinei M. (1994). «Trentacinque definizioni di etimologia ovvero : il concetto di etimologia revisitato », Quaderni di Semantica XV : 199-221.

Alinei M. (1996). «Aspetti teorici della motivazione», Quaderni di Semantica XVII : 7-17.

Alinei M. (1996a). Origini delle lingue d'Europa. I. Teoria della continuità. Bologna: Il Mulino.

Alinei M. (2000). Origini delle lingue d'Europa. II. Continuità del Mesolitico all'età del Ferro nelle principali aree etnolinguistiche. Bologna : Il Mulino.

Bartoli M. (1945). Saggi di linguistica spaziale. Torino: Rosenberg \& Sellier.

Benveniste E. (1966). Problèmes de linguistique générale, T. 1. Paris : Gallimard.

Benveniste E. (1969). Le vocabulaire des institutions indoeuropéennes. 1. économie, parenté, société. Paris : Editions de minuit.

Blank A. (2001). « Pour une approche cognitive du changement sémantique lexical : aspect sémasiologique », in J. François (éd.) Théories contemporaines du changement sémantique. Louvain \& Paris : Peeters (Mém. Soc. Ling. de Paris), 5974. 


\section{J.-P. DALBERA}

Caprini R. (2001). "Synthèse romane de la chenille», in M. Contini \& G. Tuaillon (éd.) Atlas Linguistique Roman II. Roma, IPZS, 61-87.

Caprini R. (éd.) (2003). Parole romanze. Alessandria : Edizioni dell'Orso.

Chambon J.P. \& Lüdi G. (éd.), (1991). Discours étymologiques. Tübingen : Niemeyer.

Dalbera J.Ph. (1994). Les parlers des Alpes-Maritimes. Etude comparative. Essai de reconstruction. London : AIEO.

Dalbera J.Ph. (1997). «Dimension diatopique, ressort motivationnel et étymologie. A propos des désignations romanes de l'orvet », Quaderni di Semantica XVIII : 195-21.

Dalbera J.Ph. (2002). « La géolinguistique : un nouveau souffle », Revue Belge de Philologie et d'Histoire 80(3) : 831-849.

Dalbera J.Ph. \& Dalbera-Stefanaggi M.J. (2003). « Onomasiologie, sémasiologie, étymologie. Le cas de baudroie, crapaud et autres enflures ", in J.C. Bouvier, J. Gourc, F. Pic (éd.) Sempre los camps auràn segadas resurgantas. Toulouse : Méridienne, 89-100.

Dalbera J.Ph. \& Dalbera-Stefanaggi M.J. (2003). «La petite bête qui monte, qui monte. La dimension motivationnelle dans la dénomination du petit. Etymologie et reconstruction lexicale », in R. Caprini (éd.) Parole romanze. Alessandria : Edizioni dell'Orso, 113-138.

Dalbera J.Ph. (2005). « The reproductive cycle of zoonyms », in A. Minelli, G. Ortalli, G. Sanga (éd.) Animal names. Venezia : IVSA, 293-306.

Dalbera J.Ph. (2006a). Des dialectes au langage. Une archéologie du sens. Paris : Champion.

Dalbera J.Ph. (2006b). «Zoonymes et relations parentélaires: réflexions sur la belette. De l'étymologie à la reconstruction lexicale ", Quaderni di Semantica XXVII : 225-251.

Dalbera J.Ph. (2010). «Etude des noms romans des grives, merles et autres étouneaux. Essai de reconstruction lexicale », Quaderni di Semantica XXXI/1 : 13-40. 
Dalbera J.Ph. (2012). «En amont de la phonologie diachronique du galloroman : nouvelles approches de l'étymologie et du changement lexical », in T. Scheer, P. Sauzet, M. BarraJover, J.Ph. Dalbera, G. Brun-Trigaud (éd.) Approches de la linguistique gallo-romane. Saint-Denis : PUV, 347-367.

Dalbera J.Ph. (2012). «When Dialectology studies contribute to lexical semantics and to Etymology. The contribution of the Romance language area », in J. Berns, H. Jacobs, T. Scheer (éd.) Romance Languages and Linguistic Theory. Selected papers from'Going Romance' Nice 2009. Amsterdam: Benjamins, 89-115.

Dalbera J.Ph (à paraître). « Synthèse des dénominations romanes de la grive », in M. Contini (éd.) Atlas Linguistique Roman. Roma : IPZS.

Guiraud P. (1964). L'étymologie. Paris : PUF.

Guiraud P. (1967). Structures étymologiques du lexique français. Paris : Larousse.

Guiraud P. (1982). Dictionnaire des étymologies obscures. Paris : Payot.

Koch P. (2001). « Pour une approche cognitive du changement sémantique lexical : aspect onomasiologique », in J. François (éd.) Théories contemporaines du changement sémantique. Louvain \& Paris : Peeters (Mém. Soc. Ling. de Paris), 7595.

Lamberterie Ch. de (2001). «Problèmes sémantiques de la reconstruction en indo-européen », in J. François (éd.) Théories contemporaines $d u$ changement sémantique. Louvain \& Paris : Peeters (Mém. Soc. Ling. de Paris), 109-134.

Martinet A. (1955). Economie des changements phonétiques. Berne : Francke.

Martins A.M. \& Vitorino G. (1984). «Les désignations de l'hirondelle dans les parlers romans ", Géolinguistique 1 : 129-145.

Nyckees V. (2001). « Changement de sens et déterminisme socioculturel », in J. François (éd.) Théories contemporaines $d u$ 


\section{J.-P. DALBERA}

changement sémantique. Louvain \& Paris : Peeters (Mém. Soc. Ling. de Paris), 31-58.

Rastier F. (2001). « De la sémantique cognitive à la sémantique diachronique: les valeurs et l'évolution des classes lexicales ", in J. François (éd.) Théories contemporaines $d u$ changement sémantique. Louvain \& Paris : Peeters (Mém. Soc. Ling. de Paris), 135-164.

Rousseau A. (2001). «L'évolution lexico-sémantique. Explications traditionelles et propositions nouvelles », in J. François (éd.) Théories contemporaines du changement sémantique. Louvain \& Paris : Peeters (Mém. Soc. Ling. de Paris), 1130.

Swiggers P. (1991). «Le travail étymologique : typologie historique et analytique, perspectives, effets », in J.P. Chambon, G. Lüdi (éd.) Discours étymologiques. Tübingen : Niemeyer, 29-45.

Zamboni A. (1972). «Sul friulano cidivoc », Studi mediolatini e volgare XX : 223-239. 